\title{
DYNAMIC PROPERTIES FOR THE INDUCED MAPS ON $n$-FOLD SYMMETRIC PRODUCT SUSPENSIONS
}

\author{
Franco Barragán, Alicia Santiago-Santos and Jesús F. Tenorio \\ Universidad Tecnológica de la Mixteca, México
}

\begin{abstract}
Let $X$ be a continuum. For any positive integer $n$ we consider the hyperspace $\mathcal{F}_{n}(X)$ and if $n$ is greater than or equal to two, we consider the quotient space $\mathcal{S F}_{n}(X)$ defined in [3]. For a given map $f: X \rightarrow X$, we consider the induced maps $\mathcal{F}_{n}(f): \mathcal{F}_{n}(X) \rightarrow \mathcal{F}_{n}(X)$ and $\mathcal{S F}_{n}(f): \mathcal{S F}_{n}(X) \rightarrow \mathcal{S F}_{n}(X)$ defined in [4]. Let $\mathcal{M}$ be one of the following classes of maps: exact, mixing, weakly mixing, transitive, totally transitive, strongly transitive, chaotic, minimal, irreducible, feebly open and turbulent. In this paper we study the relationships between the following statements: $f \in \mathcal{M}, \mathcal{F}_{n}(f) \in \mathcal{M}$ and $\mathcal{S F}_{n}(f) \in \mathcal{M}$.
\end{abstract}

\section{INTRODUCTION}

A continuum is a nonempty compact connected metric space. Given a continuum $X$ and a positive integer $n$, we consider the hyperspaces $2^{X}, \mathcal{C}_{n}(X)$ and $\mathcal{F}_{n}(X)$ of $X$, topologized with the Hausdorff metric. We recall that $2^{X}$ consists of all nonempty and closed subsets of $X, \mathcal{C}_{n}(X)$ consists of all elements of $2^{X}$ with at most $n$ components and $\mathcal{F}_{n}(X)$ consists of all elements of $2^{X}$ with at most $n$ points. If $n$ is an integer greater than or equal to two, by $\mathcal{S F}_{n}(X)$ we mean the quotient space $\mathcal{F}_{n}(X) / \mathcal{F}_{1}(X)$. The space $\mathcal{S F}_{n}(X)$ is called the $n$-fold symmetric product suspension of the continuum $X$. Some topological properties of $\mathcal{S F}_{n}(X)$ are studied in [3] and [5].

A map $f: X \rightarrow X$, where $X$ is a continuum, induces a map on the hyperspace $2^{X}$ denoted by $2^{f}: 2^{X} \rightarrow 2^{X}$ and defined by $2^{f}(A)=f(A)$, for each $A \in 2^{X}$. The induced map to the other hyperspaces mentioned are

2010 Mathematics Subject Classification. 54B20, 37B45, 54F50, 54F15.

Key words and phrases. Chaotic map, exact map, feebly open map, hyperspace, induced map, irreducible map, minimal map, mixing map, strongly transitive map, symmetric product, symmetric product suspension, totally transitive map, transitive map, turbulent map, weakly mixing map. 
simply the restriction of $2^{f}$ to each of such hyperspaces, denoted by $\mathcal{C}_{n}(f)$ and $\mathcal{F}_{n}(f)$, respectively, for each positive integer $n$. If $n$ is an integer greater than or equal to two, we consider the induced map $\mathcal{S F}_{n}(f): \mathcal{S F}_{n}(X) \rightarrow \mathcal{S F}_{n}(X)$, which is called induced map of $f$ on the $n$-fold symmetric product suspension of $X$. Some topological properties of $\mathcal{S F}_{n}(f)$ are studied in [4] and [6].

A dynamical system is a pair $(X, f)$, where $X$ is a continuum and $f: X \rightarrow X$ is a map. The dynamical system $(X, f)$ induce the dynamical systems $\left(2^{X}, 2^{f}\right),\left(\mathcal{C}_{n}(X), \mathcal{C}_{n}(f)\right)$ and $\left(\mathcal{F}_{n}(X), \mathcal{F}_{n}(f)\right)$. Because dynamics is obtained by iterating the map, it is important to study the dynamical properties of the map. Hence, in recent times, a natural problem has been to study connections between dynamical properties of $f$ (individual dynamics) and dynamical properties of induced maps (collective dynamics). Some dynamical properties of the induced maps $2^{f}, \mathcal{C}_{n}(f)$ and $\mathcal{F}_{n}(f)$, and others set-valued maps are studied, for instance in $[1,2,7,8,10-12,16,20,21,23]$.

In this paper, we introduce the dynamical system $\left(\mathcal{S F}_{n}(X), \mathcal{S F}_{n}(f)\right)$ and we investigate connections between dynamical properties of $f$ and the dynamical properties of the induced maps $\mathcal{F}_{n}(f)$ and $\mathcal{S F}_{n}(f)$. Specifically, if $\mathcal{M}$ is one of the following classes of maps: exact, mixing, weakly mixing, transitive, totally transitive, strongly transitive, chaotic, minimal, irreducible, feebly open and turbulent, we study the relationships between the following statements:

(1) $f \in \mathcal{M}$

(2) $\mathcal{F}_{n}(f) \in \mathcal{M}$

(3) $\mathcal{S F}_{n}(f) \in \mathcal{M}$

This paper is organized as follows: In Section 2, we recall basic definitions and introduce some notation. In Section 3, we present some preliminary results needed for the rest of the paper. In particular, we prove results respect to quotient spaces. Section 4 is devoted to study the problem posed if $\mathcal{M}$ is one of the following classes of maps: exact, mixing, weakly mixing, transitive, totally transitive, strongly transitive, chaotic and minimal. Finally, in Section 5 , we investigate our problem when $\mathcal{M}$ is one of the following classes of maps: irreducible, feebly open and turbulent.

\section{Definitions and notations}

A continuum is a nonempty compact connected metric space. A continuum is said to be nondegenerate if it has more than one point. A subcontinuum is a continuum contained in a topological space. Given a continuum $X$, a point $a \in X$ and $\epsilon>0, V_{\epsilon}(a)$ denotes the open ball with center $a$ and radius $\epsilon$. A map is a continuous function. We denote by $I d_{X}$ the identity map on a continuum $X$. A dynamical system is a pair $(X, f)$, where $X$ is a continuum and $f: X \rightarrow X$ is a map. 
The symbol $\mathbb{N}$ denote the set of positive integers. Given a dynamical system $(X, f)$, define $f^{0}=I d_{X}$ and for each $k \in \mathbb{N}$, let $f^{k}=f \circ f^{k-1}$. A point $p \in X$ is a periodic point in $(X, f)$ provided that there exists $k \in \mathbb{N}$ such that $f^{k}(p)=p$. The set of periodic points of $(X, f)$ is denoted by $\operatorname{per}(f)$. Given $x \in X$, the orbit of $x$ under $f$ is the set $\operatorname{orb}(x, f)=\left\{f^{k}(x) \mid k \in \mathbb{N} \cup\{0\}\right\}$. A subset $K$ of $X$ is said to be invariant under $f$ if $f(K) \subseteq K$ and strongly invariant under $f$ if $f(K)=K$.

Let $X$ be a continuum with metric $d$ and let $f: X \rightarrow X$ be a map. We say that $f$ is:

- exact if for each nonempty open subset $U$ of $X$, there exists $k \in \mathbb{N}$ such that $f^{k}(U)=X$;

- mixing if for every pair of nonempty open subsets $U$ and $V$ of $X$, there exists $N \in \mathbb{N}$ such that $f^{k}(U) \cap V \neq \emptyset$, for every $k \geq N$;

- weakly mixing if for all nonempty open subsets $U_{1}, U_{2}, V_{1}$ and $V_{2}$ of $X$, there exists $k \in \mathbb{N}$ such that $f^{k}\left(U_{i}\right) \cap V_{i} \neq \emptyset$, for each $i \in\{1,2\}$;

- transitive if for every pair of nonempty open subsets $U$ and $V$ of $X$, there exists $k \in \mathbb{N}$ such that $f^{k}(U) \cap V \neq \emptyset$;

- totally transitive if $f^{s}$ is transitive, for all $s \in \mathbb{N}$;

- strongly transitive if for each nonempty open subset $U$ of $X$, there exists $s \in \mathbb{N}$ such that $X=\bigcup_{k=0}^{s} f^{k}(U)$;

- chaotic if it is transitive and $\operatorname{per}(f)$ is dense in $X$;

- minimal if there is no proper subset $M \subseteq X$ which is nonempty, closed and $M$ is invariant under $f$; equivalently, if the orbit of every point of $X$ is dense in $X$;

- irreducible if the only closed subset $A \subseteq X$ for which $f(A)=X$ is $A=X$

- feebly open if for every nonempty open subset $U$ of $X$, there is a nonempty open subset $V$ of $X$ such that $V \subseteq f(U)$;

- turbulent if there are compact nondegenerate subsets $C$ and $K$ of $X$ such that $C \cap K$ has at most a point and $K \cup C \subseteq f(K) \cap f(C)$;

- isometry if $d(x, y)=d(f(x), f(y))$, for each $x, y \in X$.

Diagram 1 shows inclusions between some classes of maps, which are considered here, an arrow means inclusion; i.e., the class of maps above is contained in the class of maps below. For some of this inclusions see, for instance, [14] and [15].

Given a continuum $X$ and a positive integer $n$, we consider the following hyperspaces of $X$ :

$$
\begin{aligned}
2^{X} & =\{A \subseteq X \mid A \text { is closed and nonempty }\} \\
\mathcal{C}_{n}(X) & =\left\{A \in 2^{X} \mid A \text { has at most } n \text { components }\right\} \\
\mathcal{F}_{n}(X) & =\left\{A \in 2^{X} \mid A \text { has at most } n \text { points }\right\}
\end{aligned}
$$


We topologize these sets with the Hausdorff metric $([18,(0.1)])$. The hyperspace $\mathcal{C}_{n}(X)$ is the $n$-fold hyperspace of $X$ and the hyperspace $\mathcal{F}_{n}(X)$ is the $n$-fold symmetric product of $X$.

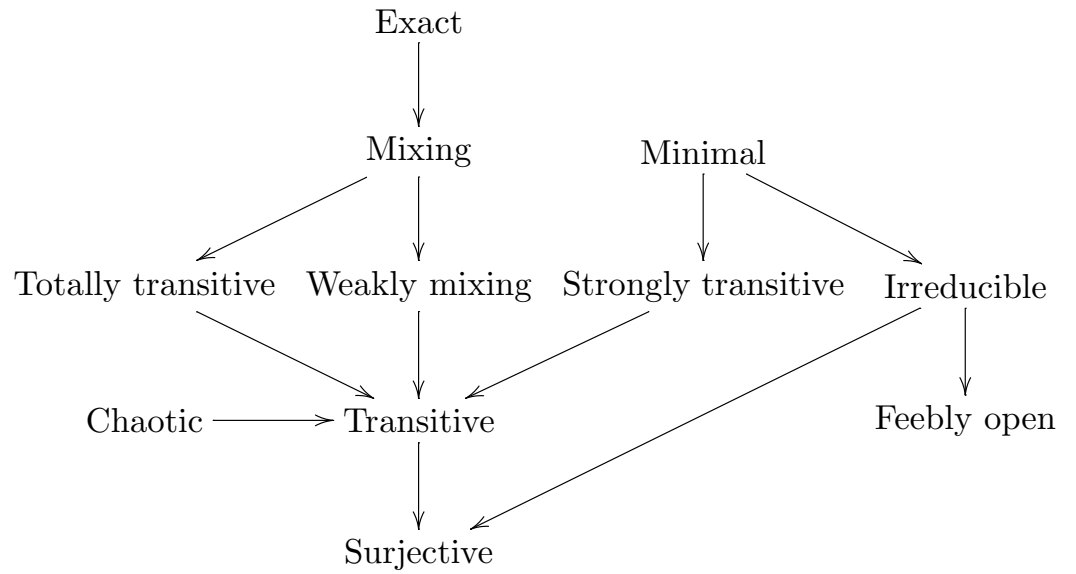

\section{DIAGRAM 1}

Given a finite collection $U_{1}, U_{2}, \ldots, U_{m}$ of nonempty subsets of $X$, we denote by $\left\langle U_{1}, U_{2}, \ldots, U_{m}\right\rangle$ the following subset of $2^{X}$ :

$$
\left\{A \in 2^{X} \mid A \subseteq \bigcup_{i=1}^{m} U_{i} \text { and } A \cap U_{i} \neq \emptyset, \text { for each } i \in\{1,2, \ldots, m\}\right\} .
$$

The family:

$$
\left\{\left\langle U_{1}, U_{2}, \ldots, U_{l}\right\rangle \mid l \in \mathbb{N} \text { and } U_{1}, U_{2}, \ldots, U_{l} \text { are open subsets of } X\right\}
$$

forms a base for a topology on $2^{X}$ called the Vietoris topology $([18,(0.11)])$. It is well known that the Vietoris topology and the topology induced by the Hausdorff metric coincide $([18,(0.13)])$. For those who are interested in learning more about this topics can see [13,17] and [19].

Notation 2.1. Let $X$ be a continuum, let $n$ be a positive integer, and let $U_{1}, U_{2}, \ldots, U_{m}$ be a finite family of open subsets of $X$. Then $\left\langle U_{1}, U_{2}, \ldots, U_{m}\right\rangle_{n}$ denotes the set $\left\langle U_{1}, U_{2}, \ldots, U_{m}\right\rangle \cap \mathcal{F}_{n}(X)$.

Let $n$ be an integer greater than or equal to two. Then the $n$-fold symmetric product suspension ([3]) of a continuum $X$, denoted by $\mathcal{S F}_{n}(X)$, is the quotient space $\mathcal{F}_{n}(X) / \mathcal{F}_{1}(X)$, with the quotient topology. Here, we denote the quotient map by $q: \mathcal{F}_{n}(X) \rightarrow \mathcal{S F}_{n}(X)$ and $q\left(\mathcal{F}_{1}(X)\right)$ by $F_{X}$. Thus,

$$
\mathcal{S F}_{n}(X)=\left\{\{A\} \mid A \in \mathcal{F}_{n}(X) \backslash \mathcal{F}_{1}(X)\right\} \cup\left\{F_{X}\right\} .
$$

REMARK 2.2. The space $\mathcal{S F}_{n}(X) \backslash\left\{F_{X}\right\}$ is homeomorphic to $\mathcal{F}_{n}(X) \backslash$ $\mathcal{F}_{1}(X)$, using the appropriate restriction of $q$. 
Let $n$ be a positive integer and let $X$ be a continuum. If $f: X \rightarrow X$ is a map, we consider the function $\mathcal{F}_{n}(f): \mathcal{F}_{n}(X) \rightarrow \mathcal{F}_{n}(X)$ defined by $\mathcal{F}_{n}(f)(A)=f(A)$, for all $A \in \mathcal{F}_{n}(X)$; it is called the induced map of $f$ on the $n$-fold symmetric product of $X$. Note that $\mathcal{F}_{n}(f)$ is continuous ([17, 1.8.23]). Also, if $n$ is greater than or equal to two, we consider the function $\mathcal{S F}_{n}(f): \mathcal{S F}_{n}(X) \rightarrow \mathcal{S F}_{n}(X)$ given by

$$
\mathcal{S F}_{n}(f)(\chi)= \begin{cases}q\left(\mathcal{F}_{n}(f)\left(q^{-1}(\chi)\right)\right), & \text { if } \chi \neq F_{X} \\ F_{X}, & \text { if } \chi=F_{X}\end{cases}
$$

Note that, by $[9,4.3$, p. 126$], \mathcal{S F}_{n}(f)$ is continuous, it is called induced map of $f$ on $n$-fold symmetric product suspension (see [4] and [6]). In addition, the diagram:

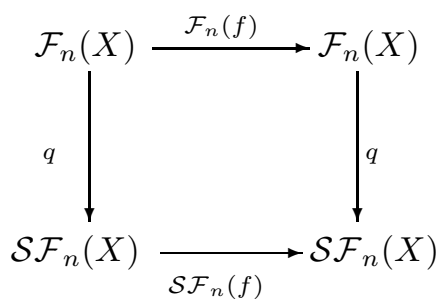

is commutative, that is $q \circ \mathcal{F}_{n}(f)=\mathcal{S F}_{n}(f) \circ q$.

As a consequence of Diagram 1 and [4, Theorem 3.2], we obtain:

Lemma 2.3. Let $X$ be a continuum, let $n$ be an integer greater than or equal to two, and let $f: X \rightarrow X$ be a map. Let $\mathcal{M}$ be one of the following classes of maps: exact, mixing, weakly mixing, transitive, totally transitive, strongly transitive, chaotic, minimal and irreducible. If $f \in \mathcal{M}$, then $f, \mathcal{F}_{n}(f)$ and $\mathcal{S F}_{n}(f)$ are surjective.

\section{Preliminary Results}

Let $X$ be a continuum, let $f: X \rightarrow X$ be a surjective map, and let $K$ be a subcontinuum of $X$ such that $K$ is strongly invariant under $f$. Consider the quotient space $X / K$ and let $q_{X}: X \rightarrow X / K$ be the quotient map. We denote $q_{X}(K)$ by $K_{X}$. Note that $f$ induces a function $f_{\star}: X / K \rightarrow X / K([9,7.7, \mathrm{p}$. 17]) given by

$$
f_{\star}(\chi)= \begin{cases}q_{X}\left(f\left(\left(q_{X}\right)^{-1}(\chi)\right)\right), & \text { if } \chi \neq K_{X} \\ K_{X}, & \text { if } \chi=K_{X} .\end{cases}
$$

The continuity of $f_{\star}$ follows from $[9,4.3$, p. 126]. Observe that the diagram 


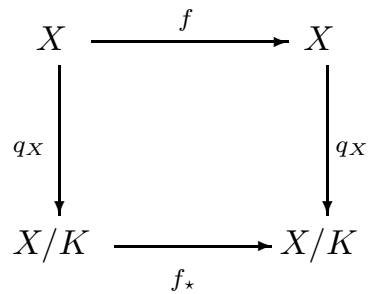

is commutative, that is $q_{X} \circ f=f_{\star} \circ q_{X}$.

Remark 3.1. Let $X$ be a continuum, let $f: X \rightarrow X$ be a surjective map, and let $K$ be a subcontinuum of $X$ such that $K$ is strongly invariant under $f$. It follows that the dynamical system $(X, f)$ induces the dynamical system $\left(X / K, f_{\star}\right)$.

As an easy consequence from the definition of $f^{k}$ and from commutativity of $(* *)$, we have the following:

Proposition 3.2. Let $X$ be a continuum, let $f: X \rightarrow X$ be a surjective map, let $K$ be a subcontinuum of $X$ such that $K$ is strongly invariant under $f$, and let $k, s \in \mathbb{N}$. Then the following holds:
(a) $q_{X} \circ f^{k}=\left(f_{\star}\right)^{k} \circ q_{X}$,
(b) $q_{X} \circ f^{k}=\left(f^{k}\right)_{\star} \circ q_{X}$,
(c) $f^{k} \circ f^{s}=f^{k+s}$,
(d) $\left(f^{s}\right)^{k}=f^{s k}$,
(e) $q_{X} \circ\left(f^{s}\right)^{k}=\left(\left(f_{\star}\right)^{s}\right)^{k} \circ q_{X}$,
(f) $q_{X} \circ\left(f^{s}\right)^{k}=\left(\left(f^{s}\right)_{\star}\right)^{k} \circ q_{X}$

Lemma 3.3. Let $X$ be a continuum, let $f: X \rightarrow X$ be a surjective map, and let $K$ be a subcontinuum of $X$ such that $K$ is strongly invariant under $f$. If $\operatorname{per}(f)$ is dense in $X$, then $\operatorname{per}\left(f_{\star}\right)$ is dense in $X / K$.

Proof. Let $\mathcal{U}$ be a nonempty open subset of $X / K$. Since $q_{X}$ is continuous, $q_{X}^{-1}(\mathcal{U})$ is a nonempty open subset of $X$. Since $\operatorname{per}(f)$ is dense in $X$, we have that $q_{X}^{-1}(\mathcal{U}) \cap \operatorname{per}(f) \neq \emptyset$. Hence, there exists $a \in q_{X}^{-1}(\mathcal{U})$ and there exists $k \in \mathbb{N}$ such that $f^{k}(a)=a$. Thus,

$$
q_{X}(a) \in \mathcal{U} \text { and } q_{X}\left(f^{k}(a)\right)=q_{X}(a) .
$$

By Proposition 3.2 (a), it follows that

$$
q_{X}(a) \in \mathcal{U} \text { and }\left(f_{\star}\right)^{k}\left(q_{X}(a)\right)=q_{X}(a) .
$$

This implies that $\mathcal{U} \cap \operatorname{per}\left(f_{\star}\right) \neq \emptyset$. Thus, we conclude that $\operatorname{per}\left(f_{\star}\right)$ is dense in $X / K$. 
Theorem 3.4. Let $X$ be a continuum, let $f: X \rightarrow X$ be a map, and let $K$ be a subcontinuum of $X$ such that $K$ is strongly invariant under $f$. Let $\mathcal{M}$ be one of the following classes of maps: exact, mixing, weakly mixing, transitive, totally transitive, strongly transitive, chaotic and minimal. If $f \in \mathcal{M}$, then $f_{\star} \in \mathcal{M}$.

Proof. Suppose that $f$ is exact, we see that $f_{\star}$ is exact. For this, let $\mathcal{U}$ be a nonempty open subset of $X / K$. Since $q_{X}$ is continuous, we have that $q_{X}^{-1}(\mathcal{U})$ is a nonempty open subset of $X$. Since $f$ is exact, there exists $k \in \mathbb{N}$ such that $f^{k}\left(q_{X}^{-1}(\mathcal{U})\right)=X$. Thus, since $q_{X}$ is surjective, we have that $q_{X}\left(f^{k}\left(q_{X}^{-1}(\mathcal{U})\right)\right)=X / K$. Hence, by Proposition 3.2 (a), we obtain that $\left(f_{\star}\right)^{k}\left(q_{X}\left(\left(q_{X}^{-1}(\mathcal{U})\right)\right)=X / K\right.$. This implies that $\left(f_{\star}\right)^{k}(\mathcal{U})=X / K$. Therefore, $f_{\star}$ is exact.

Assume that $f$ is mixing, we prove that $f_{\star}$ is mixing. Let $\mathcal{U}$ and $\mathcal{V}$ be nonempty open subsets of $X / K$. Since $q_{X}$ is continuous, it follows that $q_{X}^{-1}(\mathcal{U})$ and $q_{X}^{-1}(\mathcal{V})$ are nonempty open subsets of $X$. Since $f$ is mixing, there exists $N \in \mathbb{N}$ such that

$$
f^{k}\left(q_{X}^{-1}(\mathcal{U})\right) \cap q_{X}^{-1}(\mathcal{V}) \neq \emptyset, \text { for each } k \geq N .
$$

Let $k \geq N$. We have that there exists $a \in q_{X}^{-1}(\mathcal{U})$ such that $f^{k}(a) \in q_{X}^{-1}(\mathcal{V})$. This implies that $q_{X}(a) \in \mathcal{U}$ and $q_{X}\left(f^{k}(a)\right) \in \mathcal{V}$. Hence, by Proposition 3.2 (a), we obtain that $q_{X}(a) \in \mathcal{U}$ and $\left(f_{\star}\right)^{k}\left(q_{X}(a)\right) \in \mathcal{V}$. In consequence, $\left(f_{\star}\right)^{k}\left(q_{X}(a)\right) \in\left(f_{\star}\right)^{k}(\mathcal{U})$ and $\left(f_{\star}\right)^{k}\left(q_{X}(a)\right) \in \mathcal{V}$. Thus, $\left(f_{\star}\right)^{k}(\mathcal{U}) \cap \mathcal{V} \neq \emptyset$. Therefore, $f_{\star}$ is mixing.

To verify that if $f$ is weakly mixing, then $f_{\star}$ is weakly mixing, we use a similar argument to the proof given in the previous paragraph.

Suppose that $f$ is transitive, we prove that $f_{\star}$ is transitive. Let $\mathcal{U}$ and $\mathcal{V}$ be nonempty open subsets of $X / K$. Since $q_{X}$ is continuous, $q_{X}^{-1}(\mathcal{U})$ and $q_{X}^{-1}(\mathcal{V})$ are nonempty open subsets in $X$. Since $f$ is transitive, there exists $k \in \mathbb{N}$ such that $f^{k}\left(q_{X}^{-1}(\mathcal{U})\right) \cap q_{X}^{-1}(\mathcal{V}) \neq \emptyset$. Hence, there exists $a \in q_{X}^{-1}(\mathcal{U})$ such that $f^{k}(a) \in q_{X}^{-1}(\mathcal{V})$. It follows that, $q_{X}(a) \in \mathcal{U}$ and $q_{X}\left(f^{k}(a)\right) \in \mathcal{V}$. By Proposition 3.2 (a), we have that $q_{X}(a) \in \mathcal{U}$ and $\left(f_{\star}\right)^{k}\left(q_{X}(a)\right) \in \mathcal{V}$. In consequence, $\left(f_{\star}\right)^{k}\left(q_{X}(a)\right) \in\left(f_{\star}\right)^{k}(\mathcal{U})$ and $\left(f_{\star}\right)^{k}\left(q_{X}(a)\right) \in \mathcal{V}$. Thus, $\left(f_{\star}\right)^{k}(\mathcal{U}) \cap$ $\mathcal{V} \neq \emptyset$. Therefore, $f_{\star}$ is transitive.

Assume that $f$ is totally transitive, we prove that $f_{\star}$ is totally transitive. For this, let $s \in \mathbb{N}$ and let $\mathcal{U}$ and $\mathcal{V}$ be nonempty open subsets in $X / K$. Since $q_{X}$ is continuous, we have that $q_{X}^{-1}(\mathcal{U})$ and $q_{X}^{-1}(\mathcal{V})$ are nonempty open subsets in $X$. Since $f$ is totally transitive, $f^{s}$ is transitive. In consequence, there exists $k \in \mathbb{N}$ such that $\left(f^{s}\right)^{k}\left(q_{X}^{-1}(\mathcal{U})\right) \cap q_{X}^{-1}(\mathcal{V}) \neq \emptyset$. Thus, there exists $a \in q_{X}^{-1}(\mathcal{U})$ such that $\left(f^{s}\right)^{k}(a) \in q_{X}^{-1}(\mathcal{V})$. It follows that $q_{X}(a) \in \mathcal{U}$ and $q_{X}\left(\left(f^{s}\right)^{k}(a)\right) \in \mathcal{V}$. Hence, by Proposition $3.2(\mathrm{e}), q_{X}(a) \in \mathcal{U}$ and $\left(\left(f_{\star}\right)^{s}\right)^{k}\left(q_{X}(a)\right) \in \mathcal{V}$. This implies that:

$$
\left.\left(\left(f_{\star}\right)^{s}\right)^{k}\left(q_{X}(a)\right) \in\left(\left(f_{\star}\right)^{s}\right)^{k}(\mathcal{U}) \text { and }\left(\left(f_{\star}\right)^{s}\right)^{k}\left(q_{X}(a)\right)\right) \in \mathcal{V} .
$$


Thus, $\left(\left(f_{\star}\right)^{s}\right)^{k}(\mathcal{U}) \cap \mathcal{V} \neq \emptyset$. This proves that $\left(f_{\star}\right)^{s}$ is transitive. Therefore, $f_{\star}$ is totally transitive.

Next, suppose that $f$ is strongly transitive, we see that $f_{\star}$ is strongly transitive. Let $\mathcal{U}$ be a nonempty open subset of $X / K$. By continuity of $q_{X}$, we have that $q_{X}^{-1}(\mathcal{U})$ is a nonempty open subset of $X$. Since $f$ is strongly transitive, there exists $s \in \mathbb{N}$ such that $X=\bigcup_{k=0}^{s} f^{k}\left(q_{X}^{-1}(\mathcal{U})\right)$. Hence, since $q_{X}$ is surjective, we have that:

$$
X / K=\bigcup_{k=0}^{s} q_{X}\left(f^{k}\left(q_{X}^{-1}(\mathcal{U})\right)\right) .
$$

So, by Proposition 3.2 (a) and since $q_{X}$ is surjective, we have that:

$$
X / K=\bigcup_{k=0}^{s}\left(f_{\star}\right)^{k}(\mathcal{U}) .
$$

Therefore, $f_{\star}$ is strongly transitive.

Now, assume that $f$ is chaotic. From the previous paragraph, we have that $f_{\star}$ is transitive. Furthermore, by Lemma 3.3, we have that $\operatorname{per}\left(f_{\star}\right)$ is dense in $X / K$. This proves that $f_{\star}$ is chaotic.

Finally, suppose that $f$ is minimal, we prove that $f_{\star}$ is minimal. Let $\chi \in X / K$ and let $\mathcal{U}$ be a nonempty open subset of $X / K$. Let $x \in X$ such that $q_{X}(x)=\chi$. Since $f$ is minimal, $q_{X}^{-1}(\mathcal{U}) \cap \operatorname{orb}(x, f) \neq \emptyset$. Hence, there exist $w \in q_{X}^{-1}(\mathcal{U})$ and $k \in \mathbb{N} \cup\{0\}$ such that $w=f^{k}(x)$. Thus, $q_{X}(w) \in \mathcal{U}$ and $q_{X}(w)=q_{X}\left(f^{k}(x)\right)$. By Proposition $3.2(\mathrm{a}), q_{X}(w) \in \mathcal{U}$ and $q_{X}(w)=$ $\left(f_{\star}\right)^{k}\left(q_{X}(x)\right)$. Hence, $q_{X}(w) \in \mathcal{U}$ and $q_{X}(w) \in \operatorname{orb}\left(q_{X}(x), f_{\star}\right)$. It follows that, $\mathcal{U} \cap \operatorname{orb}\left(\chi, f_{\star}\right) \neq \emptyset$. Therefore, $f_{\star}$ is minimal.

\section{Dynamical PROPERTies Related to transitivity}

Let $X$ be a continuum, let $n$ be an integer greater than or equal to two, and let $f: X \rightarrow X$ be a map. Observe that $\mathcal{F}_{1}(X)$ is a subcontinuum of $\mathcal{F}_{n}(X)$ such that $\mathcal{F}_{1}(X)$ is strongly invariant under $\mathcal{F}_{n}(f)$. By Remark 3.1, we can consider the dynamical system $\left(\mathcal{S F}_{n}(X), \mathcal{S F}_{n}(f)\right)$.

Proposition 4.1. Let $X$ be a continuum, let $n$ be an integer greater than or equal to two, and let $f: X \rightarrow X$ be a map. Then, for each $k, s \in \mathbb{N}$, the following holds:

(a) $\left(\mathcal{F}_{n}(f)\right)^{k}(A)=f^{k}(A)$, for every $A \in \mathcal{F}_{n}(X)$,

(b) $q \circ\left(\mathcal{F}_{n}(f)\right)^{k}=\left(\mathcal{S F}_{n}(f)\right)^{k} \circ q$,

(c) $\left(\left(\mathcal{F}_{n}(f)\right)^{s}\right)^{k}=\left(\mathcal{F}_{n}(f)\right)^{s k}$,

(d) $q \circ\left(\left(\mathcal{F}_{n}(f)\right)^{s}\right)^{k}=\left(\left(\mathcal{S F}_{n}(f)\right)^{s}\right)^{k} \circ q$.

Proof. Part (a) follows directly from the definition of $\mathcal{F}_{n}(f)$, and parts (b), (c) and (d) follow from Proposition 3.2. 
Remark 4.2. Let $(X, d)$ be a continuum, let $f: X \rightarrow X$ be a map, and let $k \in \mathbb{N}$. If $f$ is an isometry, then for any $x, y \in X, d(x, y)=d\left(f^{k}(x), f^{k}(y)\right)$.

Theorem 4.3. Let $(X, d)$ be a nondegenerate continuum, let $n$ be an integer greater than or equal to two, and let $f: X \rightarrow X$ be a map. If $f$ is an isometry, then $\mathcal{S F}_{n}(f)$ is not transitive.

Proof. Suppose that $f$ is an isometry and that $\mathcal{S F}_{n}(f)$ is transitive. Let $x, y \in X$ such that $x \neq y$. Let $r=d(x, y)>0$. We define $U_{1}=$ $V_{\frac{r}{4}}(x)$ and $U_{2}=V_{\frac{r}{4}}(y)$. We consider $V_{1}$ and $V_{2}$ nonempty open subsets of $X$ such that $V_{1} \cup V_{2} \subseteq U_{1}$ and $V_{1} \cap V_{2}=\emptyset$. It follows that $\left\langle U_{1}, U_{2}\right\rangle_{n}$ and $\left\langle V_{1}, V_{2}\right\rangle_{n}$ are nonempty open subsets of $\mathcal{F}_{n}(X)$ such that $\left\langle U_{1}, U_{2}\right\rangle_{n} \cap \mathcal{F}_{1}(X)=$ $\emptyset$ and $\left\langle V_{1}, V_{2}\right\rangle_{n} \cap \mathcal{F}_{1}(X)=\emptyset$. By Remark 2.2, we have that $q\left(\left\langle U_{1}, U_{2}\right\rangle_{n}\right)$ and $q\left(\left\langle V_{1}, V_{2}\right\rangle_{n}\right)$ are nonempty open subsets of $\mathcal{S F}_{n}(X)$. Since $\mathcal{S F}_{n}(f)$ is transitive, there exists $k \in \mathbb{N}$ such that:

$$
\left(\mathcal{S F}_{n}(f)\right)^{k}\left(q\left(\left\langle U_{1}, U_{2}\right\rangle_{n}\right)\right) \cap q\left(\left\langle V_{1}, V_{2}\right\rangle_{n}\right) \neq \emptyset .
$$

By Proposition 4.1 (b), we obtain that:

$$
q\left(\left(\mathcal{F}_{n}(f)\right)^{k}\left(\left\langle U_{1}, U_{2}\right\rangle_{n}\right)\right) \cap q\left(\left\langle V_{1}, V_{2}\right\rangle_{n}\right) \neq \emptyset .
$$

Let $B \in\left(\mathcal{F}_{n}(f)\right)^{k}\left(\left\langle U_{1}, U_{2}\right\rangle_{n}\right)$ such that $q(B) \in q\left(\left\langle V_{1}, V_{2}\right\rangle_{n}\right)$. We consider $A \in\left\langle V_{1}, V_{2}\right\rangle_{n}$ such that $q(A)=q(B)$. By Remark 2.2, we have that $A=B$. Let $C \in\left\langle U_{1}, U_{2}\right\rangle_{n}$ such that $\left(\mathcal{F}_{n}(f)\right)^{k}(C)=B$. Thus, $\left(\mathcal{F}_{n}(f)\right)^{k}(C)=A$. By Proposition 4.1 (a), $f^{k}(C)=A$. Let $c_{1} \in C \cap U_{1}$ and $c_{2} \in C \cap U_{2}$. Hence, $d(x, y) \leq d\left(x, c_{1}\right)+d\left(c_{1}, c_{2}\right)+d\left(c_{2}, y\right)<\frac{r}{2}+d\left(c_{1}, c_{2}\right)$. This implies that $\frac{r}{2}<$ $d\left(c_{1}, c_{2}\right)$. On the other hand, $f^{k}\left(c_{1}\right), f^{k}\left(c_{2}\right) \in f^{k}(C) \subseteq V_{1} \cup V_{2} \subseteq U_{1}$. Thus, $d\left(f^{k}\left(c_{1}\right), f^{k}\left(c_{2}\right)\right) \leq \frac{r}{2}$. In consequence, $d\left(f^{k}\left(c_{1}\right), f^{k}\left(c_{2}\right)\right)<d\left(c_{1}, c_{2}\right)$, which is a contradiction (see Remark 4.2). Therefore, we conclude that $\mathcal{S F}_{n}(f)$ is not transitive.

By Theorem 4.3 and Diagram 1, we obtain:

THEOREM 4.4. Let $X$ be a nondegenerate continuum, let $n$ be an integer greater than or equal to two, and let $f: X \rightarrow X$ be a map. Let $\mathcal{M}$ be one of the following classes of maps: exact, mixing, weakly mixing, transitive, totally transitive, strongly transitive, chaotic and minimal. If $f$ is an isometry, then $\mathcal{S F}_{n}(f) \notin \mathcal{M}$.

Our next result follows from Theorem 3.4 and Theorem 4.4.

THEOREM 4.5. Let $X$ be a nondegenerate continuum, let $n$ be an integer greater than or equal to two, and let $f: X \rightarrow X$ be a map. Let $\mathcal{M}$ be one of the following classes of maps: exact, mixing, weakly mixing, transitive, totally transitive, strongly transitive, chaotic and minimal. If $f$ is an isometry, then $\mathcal{F}_{n}(f) \notin \mathcal{M}$. 
Let $\mathbb{Q}, \mathbb{R}$ and $\mathbb{C}$ denote the set of rational numbers, real numbers and complex numbers, respectively. We denote by $S^{1}$ the set $\left\{e^{2 \pi i \theta} \in \mathbb{C} \mid \theta \in[0,1]\right\}$.

ExAmple 4.6. Let $f: S^{1} \rightarrow S^{1}$ be the map defined by $f\left(e^{2 \pi i \theta}\right)=$ $e^{2 \pi i(\theta+\alpha)}$, where $\alpha \in \mathbb{R} \backslash \mathbb{Q}$. Let $\mathcal{M}$ be one of the following classes of maps: exact, mixing, weakly mixing, transitive, totally transitive, strongly transitive, chaotic and minimal. It is know that $f$ is an isometry. Thus, by Theorem 4.4, we have that $\mathcal{S F}_{n}(f) \notin \mathcal{M}$. Moreover, by Theorem 4.5, we obtain that $\mathcal{F}_{n}(f) \notin \mathcal{M}$. However, we have that $f$ is transitive, totally transitive, strongly transitive and minimal (see [22, p. 261]).

The following result includes [10, Theorem 13] with an alternative proof.

THEOREM 4.7. Let $X$ be a nondegenerate continuum, let $n$ be an integer greater than or equal to two, and let $f: X \rightarrow X$ be a map. Then the following are equivalent:

(1) $f$ is exact;

(2) $\mathcal{F}_{n}(f)$ is exact;

(3) $\mathcal{S F}_{n}(f)$ is exact.

Proof. Suppose that $f$ is exact, we prove that $\mathcal{F}_{n}(f)$ is exact. Let $\mathcal{U}$ be a nonempty open subset of $\mathcal{F}_{n}(X)$. We see that $\left(\mathcal{F}_{n}(f)\right)^{k}(\mathcal{U})=\mathcal{F}_{n}(X)$, for some $k \in \mathbb{N}$. By [11, Lemma 4.2], there exist nonempty open subsets $U_{1}, U_{2}$, $\ldots, U_{n}$ of $X$ such that $\left\langle U_{1}, U_{2}, \ldots, U_{n}\right\rangle_{n} \subseteq \mathcal{U}$. Thus, it is enough verify that there exists $k \in \mathbb{N}$ such that

$$
\left(\mathcal{F}_{n}(f)\right)^{k}\left(\left\langle U_{1}, U_{2}, \ldots, U_{n}\right\rangle_{n}\right)=\mathcal{F}_{n}(X) .
$$

Since $f$ is exact, for each $i \in\{1,2, \ldots, n\}$ there exists $k_{i} \in \mathbb{N}$ such that $f^{k_{i}}\left(U_{i}\right)=X$. We define $k=\max \left\{k_{1}, k_{2}, \ldots, k_{n}\right\}$. Note that, for each $i \in$ $\{1,2, \ldots, n\}, f^{k}\left(U_{i}\right)=X$.

Let $B \in \mathcal{F}_{n}(X)$. We put $B=\left\{b_{1}, b_{2}, \ldots, b_{r}\right\}$ with $r \leq n$. Define $C=\left\{b_{1}, b_{2}, \ldots, b_{r}, b_{r+1}, \ldots, b_{n}\right\}$, where $b_{r}=b_{r+1}=\cdots=b_{n}$. In consequence, for each $i \in\{1,2, \ldots, n\}, b_{i} \in f^{k}\left(U_{i}\right)$. Hence, for each $i \in\{1,2, \ldots, n\}$, let $a_{i} \in U_{i}$ such that $f^{k}\left(a_{i}\right)=b_{i}$. Define $A=\left\{a_{1}, a_{2}, \ldots, a_{n}\right\}$. It follows that $A \in\left\langle U_{1}, U_{2}, \ldots, U_{n}\right\rangle_{n}$ and $\left(\mathcal{F}_{n}(f)\right)^{k}(A)=C=B$. Thus, we obtain that $B \in\left(\mathcal{F}_{n}(f)\right)^{k}\left(\left\langle U_{1}, U_{2}, \ldots, U_{n}\right\rangle_{n}\right)$. This implies that $\mathcal{F}_{n}(X) \subseteq$ $\left(\mathcal{F}_{n}(f)\right)^{k}\left(\left\langle U_{1}, U_{2}, \ldots, U_{n}\right\rangle_{n}\right)$. Thus, $\left(\mathcal{F}_{n}(f)\right)^{k}\left(\left\langle U_{1}, U_{2}, \ldots, U_{n}\right\rangle_{n}\right)=\mathcal{F}_{n}(X)$. Therefore, $\mathcal{F}_{n}(f)$ is exact.

It follows from Theorem 3.4 that if $\mathcal{F}_{n}(f)$ is exact, then $\mathcal{S F}_{n}(f)$ is exact.

Finally, assume that $\mathcal{S F}_{n}(f)$ is exact, we see that $f$ is exact. For this end, let $U$ be a nonempty open subset of $X$. We consider two open subsets $U_{1}$ and $U_{2}$ of $X$ such that $U_{1} \cup U_{2} \subseteq U$ and $U_{1} \cap U_{2}=\emptyset$. It follows that $\left\langle U_{1}, U_{2}\right\rangle_{n}$ is a nonempty open subset of $\mathcal{F}_{n}(X)$ such that $\left\langle U_{1}, U_{2}\right\rangle_{n} \cap \mathcal{F}_{1}(X)=$ $\emptyset$. Thus, by Remark 2.2 , we have that $q\left(\left\langle U_{1}, U_{2}\right\rangle_{n}\right)$ is a nonempty open subset of $\mathcal{S F}_{n}(X)$. Since $\mathcal{S F}_{n}(f)$ is exact, there exists $k \in \mathbb{N}$ such that 
$\left(\mathcal{S F}_{n}(f)\right)^{k}\left(q\left(\left\langle U_{1}, U_{2}\right\rangle_{n}\right)\right)=\mathcal{S F}_{n}(X)$. By Proposition 4.1 (b), we obtain that $q\left(\left(\mathcal{F}_{n}(f)\right)^{k}\left(\left\langle U_{1}, U_{2}\right\rangle_{n}\right)\right)=\mathcal{S F}_{n}(X)$.

Now, let $x \in X$. We consider a point $y \in X$ such that $x \neq y$ and define $A=\{x, y\}$. Note that $A \in \mathcal{F}_{n}(X) \backslash \mathcal{F}_{1}(X)$. Thus, $q(A) \neq F_{X}$. Since $q(A) \in \mathcal{S F}_{n}(X)$, there exists $B \in\left(\mathcal{F}_{n}(f)\right)^{k}\left(\left\langle U_{1}, U_{2}\right\rangle_{n}\right)$ such that $q(B)=q(A)$. Hence, by Remark 2.2 , we obtain that $B=A$. Let $C \in\left\langle U_{1}, U_{2}\right\rangle_{n}$ such that $\left(\mathcal{F}_{n}(f)\right)^{k}(C)=B$. By Proposition 4.1 (a), we deduce that $f^{k}(C)=B$. Since $A=B$ and $C \subseteq U$, it follows that $x \in f^{k}(U)$. Thus, $X \subseteq f^{k}(U)$. In consequence, $f^{k}(U)=X$. Therefore, $f$ is exact.

Our next result follows from Theorem 4.7 and Diagram 1 (compare with [7, Proposition 3.3]).

Corollary 4.8. Let $X$ be a nondegenerate continuum, let $n$ be an integer greater than or equal to two, and let $f: X \rightarrow X$ be a map. Then the following statements hold.

(1) If $f$ is exact, then $\mathcal{F}_{n}(f)$ is transitive.

(2) If $f$ is exact, then $\mathcal{S F}_{n}(f)$ is transitive.

THEOREM 4.9. Let $X$ be a continuum, let $n$ be an integer greater than or equal to two, and let $f: X \rightarrow X$ be a map. Then the following are equivalent:

(1) $f$ is mixing;

(2) $\mathcal{F}_{n}(f)$ is mixing;

(3) $\mathcal{S F}_{n}(f)$ is mixing.

Proof. As a consequence from [11, Theorem 4.3], we have that (1) implies (2); and by Theorem 3.4, it follows that (2) implies (3).

Finally, suppose that $\mathcal{S F}_{n}(f)$ is mixing, we see that $f$ is mixing. Let $U$ and $V$ be nonempty open subsets of $X$. We consider nonempty open subsets $U_{1}, U_{2}, V_{1}$ and $V_{2}$ of $X$ such that $U_{1} \cup U_{2} \subseteq U, V_{1} \cup V_{2} \subseteq V$, $U_{1} \cap U_{2}=\emptyset$ and $V_{1} \cap V_{2}=\emptyset$. Hence, $\left\langle U_{1}, U_{2}\right\rangle_{n}$ and $\left\langle V_{1}, V_{2}\right\rangle_{n}$ are nonempty open subsets of $\mathcal{F}_{n}(X)$ such that $\left\langle U_{1}, U_{2}\right\rangle_{n} \cap \mathcal{F}_{1}(X)=\emptyset$ and $\left\langle V_{1}, V_{2}\right\rangle_{n} \cap$ $\mathcal{F}_{1}(X)=\emptyset$. By Remark 2.2, $q\left(\left\langle U_{1}, U_{2}\right\rangle_{n}\right)$ and $q\left(\left\langle V_{1}, V_{2}\right\rangle_{n}\right)$ are nonempty open subsets of $\mathcal{S F}_{n}(X)$ such that $F_{X} \notin q\left(\left\langle U_{1}, U_{2}\right\rangle_{n}\right)$ and $F_{X} \notin q\left(\left\langle V_{1}, V_{2}\right\rangle_{n}\right)$. Since $\mathcal{S F}_{n}(f)$ is mixing, there exists $N \in \mathbb{N}$ such that for each $k \geq N$, $(\mathcal{S F}(f))^{k}\left(q\left(\left\langle U_{1}, U_{2}\right\rangle_{n}\right)\right) \cap q\left(\left\langle V_{1}, V_{2}\right\rangle_{n}\right) \neq \emptyset$. Fix $k \geq N$ and let $\chi \in q\left(\left\langle U_{1}, U_{2}\right\rangle_{n}\right)$ such that $\left(\mathcal{S} \mathcal{F}_{n}(f)\right)^{k}(\chi) \in q\left(\left\langle V_{1}, V_{2}\right\rangle_{n}\right)$. Let $A \in\left\langle U_{1}, U_{2}\right\rangle_{n}$ such that $q(A)=$ $\chi$ and let $B \in\left\langle V_{1}, V_{2}\right\rangle_{n}$ such that $\left(\mathcal{S F}_{n}(f)\right)^{k}(\chi)=q(B)$. It follows that $\left(\mathcal{S F}_{n}(f)\right)^{k}(q(A))=q(B)$. In consequence, by Proposition 4.1 (b), we have that $q\left(\left(\mathcal{F}_{n}(f)\right)^{k}(A)\right)=q(B)$. Hence, by Remark $2.2,\left(\mathcal{F}_{n}(f)\right)^{k}(A)=B$. Now, by Proposition 4.1 (a), we have that $f^{k}(A)=B$. Let $a \in A \cap U_{1}$. Thus, $f^{k}(a) \in f^{k}(A)$. Since $A \subseteq U$ and $B \subseteq V$, we have that $a \in U$ and $f^{k}(a) \in V$. Hence, $f^{k}(U) \cap V \neq \emptyset$. Therefore, $f$ is mixing. 
THEOREM 4.10. Let $X$ be a nondegenerate continuum, let $n$ be an integer greater than or equal to two, and let $f: X \rightarrow X$ be a map. Consider the following statements:

(1) $f$ is transitive;

(2) $\mathcal{F}_{n}(f)$ is transitive;

(3) $\mathcal{S F}_{n}(f)$ is transitive.

Then (2) and (3) are equivalent, (2) implies (1), (3) implies (1), (1) does not imply (2) and (1) does not imply (3).

Proof. As a consequence from Theorem 3.4, we have that (2) implies

Suppose that $\mathcal{S F}_{n}(f)$ is transitive, we prove that $\mathcal{F}_{n}(f)$ is transitive. To this end, let $\mathcal{U}$ and $\mathcal{V}$ be nonempty open subsets of $\mathcal{F}_{n}(X)$. By [11, Lemma 4.2], there exist nonempty open subsets $U_{1}, U_{2}, \ldots, U_{n}$ and $V_{1}, V_{2}, \ldots, V_{n}$ of $X$ such that

$$
\left\langle U_{1}, U_{2}, \ldots, U_{n}\right\rangle_{n} \subseteq \mathcal{U} \text { and }\left\langle V_{1}, V_{2}, \ldots, V_{n}\right\rangle_{n} \subseteq \mathcal{V} .
$$

For each $i \in\{1,2, \ldots, n\}$, let $W_{i}$ be a nonempty open subset of $X$ such that $W_{i} \subseteq U_{i}$ and for each $i, j \in\{1,2, \ldots, n\}, W_{i} \cap W_{j}=\emptyset$, if $i \neq j$. Similarly, for each $i \in\{1,2, \ldots, n\}$, let $O_{i}$ be a nonempty open subset of $X$ such that $O_{i} \subseteq V_{i}$ and for each $i, j \in\{1,2, \ldots, n\}, O_{i} \cap O_{j}=\emptyset$, if $i \neq j$. Note that $\left\langle U_{1}, U_{2}, \ldots, U_{n}\right\rangle_{n}$ and $\left\langle V_{1}, V_{2}, \ldots, V_{n}\right\rangle_{n}$ are nonempty open subsets of $\mathcal{F}_{n}(X)$ such that $\left\langle W_{1}, W_{2}, \ldots, W_{n}\right\rangle_{n} \subseteq\left\langle U_{1}, U_{2}, \ldots, U_{n}\right\rangle_{n} \subseteq \mathcal{U}$, $\left\langle O_{1}, O_{2}, \ldots, O_{n}\right\rangle_{n} \subseteq\left\langle V_{1}, V_{2}, \ldots, V_{n}\right\rangle_{n} \subseteq \mathcal{V},\left\langle W_{1}, W_{2}, \ldots, W_{n}\right\rangle_{n} \cap \mathcal{F}_{1}(X)=\emptyset$ and $\left\langle O_{1}, O_{2}, \ldots, O_{n}\right\rangle_{n} \cap \mathcal{F}_{1}(X)=\emptyset$. By Remark 2.2, $q\left(\left\langle W_{1}, W_{2}, \ldots, W_{n}\right\rangle_{n}\right)$ and $q\left(\left\langle O_{1}, O_{2}, \ldots, O_{n}\right\rangle_{n}\right)$ are nonempty open subsets of $\mathcal{S F}_{n}(X)$ such that $F_{X} \notin q\left(\left\langle W_{1}, W_{2}, \ldots, W_{n}\right\rangle_{n}\right)$ and $F_{X} \notin q\left(\left\langle O_{1}, O_{2}, \ldots, O_{n}\right\rangle_{n}\right)$. Since $\mathcal{S F}_{n}(f)$ is transitive, there exists $k \in \mathbb{N}$ such that:

$$
\left(\left(\mathcal{S F}_{n}(f)\right)^{k}\left(q\left(\left\langle W_{1}, W_{2}, \ldots, W_{n}\right\rangle_{n}\right)\right) \cap q\left(\left\langle O_{1}, O_{2}, \ldots, O_{n}\right\rangle_{n}\right) \neq \emptyset .\right.
$$

By Proposition 4.1 (b), it follows that:

$$
q\left(\left(\mathcal{F}_{n}(f)\right)^{k}\left(\left\langle W_{1}, W_{2}, \ldots, W_{n}\right\rangle_{n}\right)\right) \cap q\left(\left\langle O_{1}, O_{2}, \ldots, O_{n}\right\rangle_{n}\right) \neq \emptyset .
$$

From Remark 2.2, we obtain that:

$$
\left(\mathcal{F}_{n}(f)\right)^{k}\left(\left\langle W_{1}, W_{2}, \ldots, W_{n}\right\rangle_{n}\right) \cap\left\langle O_{1}, O_{2}, \ldots, O_{n}\right\rangle_{n} \neq \emptyset .
$$

This implies that $\left(\mathcal{F}_{n}(f)\right)^{k}(\mathcal{U}) \cap \mathcal{V} \neq \emptyset$. Therefore, $\mathcal{F}_{n}(f)$ is transitive.

On the other hand, by [11, Theorem 4.5] and Diagram 1, it follows that (2) implies (1). Moreover, since (2) and (3) are equivalent, we obtain that (3) implies (1).

Finally, by Example 4.6, we deduce that (1) does not imply (2) and that (1) does not imply (3). 
ThEOREM 4.11. Let $X$ be a nondegenerate continuum, let $n$ be an integer greater than or equal to two, and let $f: X \rightarrow X$ be a map. Then the following are equivalent:

(1) $f$ is weakly mixing;

(2) $\mathcal{F}_{n}(f)$ is weakly mixing;

(3) $\mathcal{F}_{n}(f)$ is transitive;

(4) $\mathcal{S F}_{n}(f)$ is weakly mixing;

(5) $\mathcal{S F}_{n}(f)$ is transitive.

Proof. By [11, Theorem 4.5], we have that (1), (2) and (3) are equivalent and by Theorem 4.10, it follows that (3) and (5) are equivalent. By Diagram 1 , we obtain that (4) implies (5). Moreover, by Theorem 3.4, we conclude that (2) implies (4). Therefore, for complete the proof of the theorem it suffices to prove that (5) implies (1).

Suppose that $\mathcal{S F}_{n}(f)$ is transitive, we prove that $f$ is weakly mixing. For this, let $U, V_{1}$ and $V_{2}$ be nonempty open subsets of $X$. By [11, Theorem 4.4], it suffices to show that there exists $k \in \mathbb{N}$ such that $f^{k}(U) \cap V_{1} \neq \emptyset$ and $f^{k}(U) \cap V_{2} \neq \emptyset$. For this end, let $U_{1}$ and $U_{2}$ be nonempty open subsets of $X$ such that $U_{1} \cap U_{2}=\emptyset$ and $U_{1} \cup U_{2} \subseteq U$. On the other hand, let $W_{1}$ and $W_{2}$ be nonempty open subsets of $X$ such that $W_{1} \subseteq V_{1}, W_{2} \subseteq$ $V_{2}$ and $W_{1} \cap W_{2}=\emptyset$. Note that $\left\langle U_{1}, U_{2}\right\rangle_{n}$ and $\left\langle W_{1}, W_{2}\right\rangle_{n}$ are nonempty open subsets of $\mathcal{F}_{n}(X)$ such that $\left\langle U_{1}, U_{2}\right\rangle_{n} \cap \mathcal{F}_{1}(X)=\emptyset$ and $\left\langle W_{1}, W_{2}\right\rangle_{n} \cap$ $\mathcal{F}_{1}(X)=\emptyset$. By Remark 2.2, it follows that $q\left(\left\langle U_{1}, U_{2}\right\rangle_{n}\right)$ and $q\left(\left\langle W_{1}, W_{2}\right\rangle_{n}\right)$ are nonempty open subsets of $\mathcal{S F}_{n}(X)$ such that $F_{X} \notin q\left(\left\langle U_{1}, U_{2}\right\rangle_{n}\right)$ and $F_{X} \notin q\left(\left\langle W_{1}, W_{2}\right\rangle_{n}\right)$. Since $\mathcal{S F}_{n}(f)$ is transitive, there exists $k \in \mathbb{N}$ such that $(\mathcal{S} \mathcal{F}(f))^{k}\left(q\left(\left\langle U_{1}, U_{2}\right\rangle_{n}\right)\right) \cap q\left(\left\langle W_{1}, W_{2}\right\rangle_{n}\right) \neq \emptyset$. Let $\chi \in q\left(\left\langle U_{1}, U_{2}\right\rangle_{n}\right)$ such that $\left(\mathcal{S F}_{n}(f)\right)^{k}(\chi) \in q\left(\left\langle W_{1}, W_{2}\right\rangle_{n}\right)$. Let $A \in\left\langle U_{1}, U_{2}\right\rangle_{n}$ such that $q(A)=\chi$ and let $B \in\left\langle W_{1}, W_{2}\right\rangle_{n}$ such that $q(B)=\left(\mathcal{S F}_{n}(f)\right)^{k}(\chi)$. This implies that $q(B)=\left(\mathcal{S F}_{n}(f)\right)^{k}(q(A))$. By Proposition $4.1(\mathrm{~b}), q(B)=q\left(\left(\mathcal{F}_{n}(f)\right)^{k}(A)\right)$. Since $q(B) \neq F_{X}$, we have that $\left(\mathcal{F}_{n}(f)\right)^{k}(A) \in \mathcal{F}_{n}(X) \backslash \mathcal{F}_{1}(X)$. Moreover, $A \in \mathcal{F}_{n}(X) \backslash \mathcal{F}_{1}(X)$. In consequence, by Remark 2.2, it follows that $B=$ $\left(\mathcal{F}_{n}(f)\right)^{k}(A)$. Hence, by Proposition 4.1 (a), we obtain that $f^{k}(A)=B$.

Now, since $B \in\left\langle W_{1}, W_{2}\right\rangle_{n}$, we have that $B \cap W_{1} \neq \emptyset$. Let $b \in B \cap W_{1}$. Thus, $b \in V_{1}$ and $b \in f^{k}(A)$. In consequence, there exists $a \in A$ such that $f^{k}(a)=b$. Furthermore, since $a \in U$, we have that $b \in f^{k}(U) \cap V_{1}$. Thus, $f^{k}(U) \cap V_{1} \neq \emptyset$. With a similar argument, we obtain that $f^{k}(U) \cap V_{2} \neq \emptyset$. Hence, by [11, Theorem 4.4], we conclude that $f$ is weakly mixing.

THEOREM 4.12. Let $X$ be a continuum, let $n$ be an integer greater than or equal to two, and let $f: X \rightarrow X$ be a map. Consider the following statements:

(1) $f$ is totally transitive;

(2) $\mathcal{F}_{n}(f)$ is totally transitive;

(3) $\mathcal{S F}_{n}(f)$ is totally transitive. 
Then (2) and (3) are equivalents, (3) implies (1), (2) implies (1), (1) does not imply (2) and (1) does not imply (3).

Proof. By Theorem 3.4, we have that (2) implies (3).

Suppose that $\mathcal{S F}_{n}(f)$ is totally transitive, we prove that $\mathcal{F}_{n}(f)$ is totally transitive. For this end, let $s \in \mathbb{N}$. We see that $\left(\mathcal{F}_{n}(f)\right)^{s}$ is transitive. Let $\mathcal{U}$ and $\mathcal{V}$ be nonempty open subsets of $\mathcal{F}_{n}(X)$. By [11, Lemma 4.2], there exist nonempty open subsets $U_{1}, U_{2}, \ldots, U_{n}$ and $V_{1}, V_{2}, \ldots, V_{n}$ of $X$ such that $\left\langle U_{1}, U_{2}, \ldots, U_{n}\right\rangle_{n} \subseteq \mathcal{U}$ and $\left\langle V_{1}, V_{2}, \ldots, V_{n}\right\rangle_{n} \subseteq \mathcal{V}$. For each $i \in\{1,2, \ldots, n\}$, let $W_{i}$ be a nonempty open subset of $X$ such that $W_{i} \subseteq U_{i}$ and for each $i, j \in\{1,2, \ldots, n\}, W_{i} \cap W_{j}=\emptyset$, if $i \neq j$. Also, for each $i \in\{1,2, \ldots, n\}$, let $O_{i}$ be a nonempty open subset of $X$ such that $O_{i} \subseteq V_{i}$ and for each $i, j \in\{1,2, \ldots, n\}, O_{i} \cap O_{j}=\emptyset$, if $i \neq j$. Note that $\left\langle U_{1}, U_{2}, \ldots, U_{n}\right\rangle_{n}$ and $\left\langle V_{1}, V_{2}, \ldots, V_{n}\right\rangle_{n}$ are nonempty open subsets of $\mathcal{F}_{n}(X)$ such that:

$$
\left\langle W_{1}, W_{2}, \ldots, W_{n}\right\rangle_{n} \subseteq\left\langle U_{1}, U_{2}, \ldots, U_{n}\right\rangle_{n} \subseteq \mathcal{U}
$$

and

$$
\left\langle O_{1}, O_{2}, \ldots, O_{n}\right\rangle_{n} \subseteq\left\langle V_{1}, V_{2}, \ldots, V_{n}\right\rangle_{n} \subseteq \mathcal{V} .
$$

Note that $\left\langle W_{1}, W_{2}, \ldots, W_{n}\right\rangle_{n} \cap \mathcal{F}_{1}(X)=\emptyset$ and $\left\langle O_{1}, O_{2}, \ldots, O_{n}\right\rangle_{n} \cap \mathcal{F}_{1}(X)=\emptyset$. Hence, by Remark 2.2, we have that:

$$
q\left(\left\langle W_{1}, W_{2}, \ldots, W_{n}\right\rangle_{n}\right) \text { and } q\left(\left\langle O_{1}, O_{2}, \ldots, O_{n}\right\rangle_{n}\right)
$$

are open subsets of $\mathcal{S F}_{n}(X)$ such that $F_{X} \notin q\left(\left\langle W_{1}, W_{2}, \ldots, W_{n}\right\rangle_{n}\right)$ and $F_{X} \notin q\left(\left\langle O_{1}, O_{2}, \ldots, O_{n}\right\rangle_{n}\right)$. Since $\mathcal{S F}_{n}(f)$ is totally transitive, $\left(\mathcal{S} \mathcal{F}_{n}(f)\right)^{s}$ is transitive. Thus, there exists $k \in \mathbb{N}$ such that:

$$
\left(\left(\mathcal{S F}_{n}(f)\right)^{s}\right)^{k}\left(q\left(\left\langle W_{1}, W_{2}, \ldots, W_{n}\right\rangle_{n}\right)\right) \cap q\left(\left\langle O_{1}, O_{2}, \ldots, O_{n}\right\rangle_{n}\right) \neq \emptyset .
$$

By Proposition 4.1 (d), it follows that:

$$
q\left(\left(\left(\mathcal{F}_{n}(f)\right)^{s}\right)^{k}\left(\left\langle W_{1}, W_{2}, \ldots, W_{n}\right\rangle_{n}\right)\right) \cap q\left(\left\langle O_{1}, O_{2}, \ldots, O_{n}\right\rangle_{n}\right) \neq \emptyset .
$$

We take $B \in\left(\left(\mathcal{F}_{n}(f)\right)^{s}\right)^{k}\left(\left\langle W_{1}, W_{2}, \ldots, W_{n}\right\rangle_{n}\right)$ with the following property: $q(B) \in q\left(\left\langle O_{1}, O_{2}, \ldots, O_{n}\right\rangle_{n}\right)$. Hence, we consider $A \in\left\langle O_{1}, O_{2}, \ldots, O_{n}\right\rangle_{n}$ such that $q(A)=q(B)$. By Remark 2.2, we obtain that $A=B$. Thus, it follows that:

$$
\left.\left(\mathcal{F}_{n}(f)\right)^{s}\right)^{k}\left(\left\langle W_{1}, W_{2}, \ldots, W_{n}\right\rangle_{n}\right) \cap\left\langle O_{1}, O_{2}, \ldots, O_{n}\right\rangle_{n} \neq \emptyset .
$$

This implies that $\left(\left(\mathcal{F}_{n}(f)\right)^{s}\right)^{k}(\mathcal{U}) \cap \mathcal{V} \neq \emptyset$. In consequence, $\left(\mathcal{F}_{n}(f)\right)^{s}$ is transitive. Therefore, $\mathcal{F}_{n}(f)$ is totally transitive.

Now, suppose that $\mathcal{S F}_{n}(f)$ is totally transitive, we see that $f$ is totally transitive. Fix $s \in \mathbb{N}$. We prove that $f^{s}$ is transitive. For this, let $U$ and $V$ be nonempty open subsets of $X$. We consider $U_{1}, U_{2}, V_{1}$ and $V_{2}$ nonempty open subsets of $X$ such that $U_{1} \cup U_{2} \subseteq U, U_{1} \cap U_{2}=\emptyset, V_{1} \cup V_{2} \subseteq V$ and $V_{1} \cap V_{2}=\emptyset$. Hence, $\left\langle U_{1}, U_{2}\right\rangle_{n}$ and $\left\langle V_{1}, V_{2}\right\rangle_{n}$ are nonempty open subsets of $\mathcal{F}_{n}(X)$ such that $\left\langle U_{1}, U_{2}\right\rangle_{n} \cap \mathcal{F}_{1}(X)=\emptyset$ and $\left\langle V_{1}, V_{2}\right\rangle_{n} \cap \mathcal{F}_{1}(X)=\emptyset$. By Remark 2.2, we obtain that $q\left(\left\langle U_{1}, U_{2}\right\rangle_{n}\right)$ and $q\left(\left\langle V_{1}, V_{2}\right\rangle_{n}\right)$ are nonempty open subsets of $\mathcal{S F}_{n}(X)$ 
such that $F_{X} \notin q\left(\left\langle U_{1}, U_{2}\right\rangle_{n}\right)$ and $F_{X} \notin q\left(\left\langle V_{1}, V_{2}\right\rangle_{n}\right)$. Since $\mathcal{S} \mathcal{F}_{n}(f)$ is totally transitive, $\left(\mathcal{S F}_{n}(f)\right)^{s}$ is transitive, in consequence, there exists $k \in \mathbb{N}$ such that:

$$
\left(\left(\mathcal{S F}_{n}(f)\right)^{s}\right)^{k}\left(q\left(\left\langle U_{1}, U_{2}\right\rangle_{n}\right)\right) \cap q\left(\left\langle V_{1}, V_{2}\right\rangle_{n}\right) \neq \emptyset .
$$

By Proposition 4.1 (d), it follows that:

$$
q\left(\left(\left(\mathcal{F}_{n}(f)\right)^{s}\right)^{k}\left(\left\langle U_{1}, U_{2}\right\rangle_{n}\right)\right) \cap q\left(\left\langle V_{1}, V_{2}\right\rangle_{n}\right) \neq \emptyset .
$$

Let $B \in\left(\left(\mathcal{F}_{n}(f)\right)^{s}\right)^{k}\left(\left\langle U_{1}, U_{2}\right\rangle_{n}\right)$ such that $q(B) \in q\left(\left\langle V_{1}, V_{2}\right\rangle_{n}\right)$. We consider an element $A \in\left\langle V_{1}, V_{2}\right\rangle_{n}$ such that $q(B)=q(A)$. By Remark 2.2, we obtain that $A=B$. This implies that:

$$
\left(\left(\mathcal{F}_{n}(f)\right)^{s}\right)^{k}\left(\left\langle U_{1}, U_{2}\right\rangle_{n}\right) \cap\left\langle V_{1}, V_{2}\right\rangle_{n} \neq \emptyset .
$$

Let $C \in\left\langle U_{1}, U_{2}\right\rangle_{n}$ such that $\left(\left(\mathcal{F}_{n}(f)\right)^{s}\right)^{k}(C) \in\left\langle V_{1}, V_{2}\right\rangle_{n}$. We consider $D \in$ $\left\langle V_{1}, V_{2}\right\rangle_{n}$ such that $\left(\left(\mathcal{F}_{n}(f)\right)^{s}\right)^{k}(C)=D$. By Proposition 4.1 (c), we have that $\left(\mathcal{F}_{n}(f)\right)^{s k}(C)=D$. Thus, by Proposition 4.1 (a), $f^{s k}(C)=D$. Now, we consider an element $a \in C \cap U_{1}$. It follows that $f^{s k}(a) \in D$. Since $C \subseteq U$ and $D \subseteq V$, we have that $f^{s k}(a) \in f^{s k}(U) \cap V$. By Proposition 3.2 (d), $\left(f^{s}\right)^{k}(a) \in\left(f^{s}\right)^{k}(U) \cap V$. Hence, $\left(f^{s}\right)^{k}(U) \cap V \neq \emptyset$. In consequence, $f^{s}$ is transitive. Therefore, $f$ is totally transitive.

On the other hand, since (2) implies (3) and (3) implies (1), we obtain that (2) implies (1).

Finally, by Example 4.6, we see that (1) does not imply (2) and (1) does not imply (3).

TheOREM 4.13. Let $X$ be a nondegenerate continuum, let $n$ be an integer greater than or equal to two, and let $f: X \rightarrow X$ be a map. Consider the following statements:

(1) $f$ is strongly transitive;

(2) $\mathcal{F}_{n}(f)$ is strongly transitive;

(3) $\mathcal{S F}_{n}(f)$ is strongly transitive.

Then (2) implies (3), (3) implies (1), (2) implies (1), (1) does not imply (2) and (1) does not imply (3).

Proof. By Theorem 3.4, we have that (2) implies (3).

Now, suppose that $\mathcal{S F}_{n}(f)$ is strongly transitive, we see that $f$ is strongly transitive. Let $U$ be a nonempty open subset of $X$. We consider $U_{1}$ and $U_{2}$ nonempty open subsets of $X$ such that $U_{1} \cup U_{2} \subseteq U$ and $U_{1} \cap U_{2}=\emptyset$. It follows that $\left\langle U_{1}, U_{2}\right\rangle_{n}$ is a nonempty open subset of $\mathcal{F}_{n}(X)$ such that $\left\langle U_{1}, U_{2}\right\rangle_{n} \cap$ $\mathcal{F}_{1}(X)=\emptyset$. By Remark 2.2, we have that $q\left(\left\langle U_{1}, U_{2}\right\rangle_{n}\right)$ is a nonempty open subset of $\mathcal{S F}_{n}(X)$ such that $F_{X} \notin q\left(\left\langle U_{1}, U_{2}\right\rangle_{n}\right)$. Since $\mathcal{S F}_{n}(f)$ is strongly transitive, there exists $s \in \mathbb{N}$ such that:

$$
\mathcal{S F}_{n}(X)=\bigcup_{k=0}^{s}\left(\mathcal{S F}_{n}(f)\right)^{k}\left(q\left(\left\langle U_{1}, U_{2}\right\rangle_{n}\right)\right) .
$$


By Proposition 4.1 (b), we obtain that:

$$
\mathcal{S} \mathcal{F}_{n}(X)=\bigcup_{k=0}^{s} q\left(\left(\mathcal{F}_{n}(f)\right)^{k}\left(\left\langle U_{1}, U_{2}\right\rangle_{n}\right)\right) \text {. }
$$

We prove that $X=\bigcup_{k=0}^{s} f^{k}(U)$. For this end, let $x \in X$. We fix $y \in X \backslash\{x\}$ and we consider $A=\{x, y\}$. Thus, $A \in \mathcal{F}_{n}(X) \backslash \mathcal{F}_{1}(X)$. In consequence, $q(A) \in \mathcal{S F}_{n}(X) \backslash\left\{F_{X}\right\}$. This implies that there exists $j \in$ $\{0,1, \ldots, s\}$ such that $q(A) \in q\left(\left(\mathcal{F}_{n}(f)\right)^{j}\left(\left\langle U_{1}, U_{2}\right\rangle_{n}\right)\right)$. Hence, there exists $B \in$ $\left(\mathcal{F}_{n}(f)\right)^{j}\left(\left\langle U_{1}, U_{2}\right\rangle_{n}\right)$ such that $q(B)=q(A)$. Note that, by Remark 2.2, $A=$ $B$. On the other hand, there exists $C \in\left\langle U_{1}, U_{2}\right\rangle_{n}$ such that $\left(\mathcal{F}_{n}(f)\right)^{j}(C)=B$. By Proposition 4.1 (a), $f^{j}(C)=B$. Moreover, since $C \subseteq U$, it follows that $A \subseteq f^{j}(U)$. In consequence, $x \in f^{j}(U)$. Thus, $X \subseteq \bar{\bigcup}_{k=0}^{s} f^{k}(U)$. Hence, $X=\bigcup_{k=0}^{s} f^{k}(U)$. Therefore, $f$ is strongly transitive.

Since (2) implies (3) and (3) implies (1), we obtain that (2) implies (1).

Finally, in Example 4.6, we have that (1) does not implies (2) and that (1) does not implies (3).

As a consequence of Diagram 1 and Theorem 4.10, we have the follows:

Corollary 4.14. Let $X$ be a nondegenerate continuum, let $n$ be an integer greater than or equal to two, and let $f: X \rightarrow X$ be a map. If $\mathcal{S F}_{n}(f)$ is strongly transitive, then $\mathcal{F}_{n}(f)$ is transitive.

Moreover, by Corollary 4.14 and Theorem 4.11, we obtain:

Corollary 4.15. Let $X$ be a nondegenerate continuum, let $n$ be an integer greater than or equal to two, and let $f: X \rightarrow X$ be a map. If $\mathcal{S F}_{n}(f)$ is strongly transitive, then $f, \mathcal{F}_{n}(f)$ and $\mathcal{S F}_{n}(f)$ are weakly mixing.

QUESTION 4.1. Let $X$ be a continuum, let $n$ be an integer greater than or equal to two, and let $f: X \rightarrow X$ be a map. If $\mathcal{S F}_{n}(f)$ is strongly transitive, then is $\mathcal{F}_{n}(f)$ strongly transitive?

The following lemma is used in the proof of Theorem 4.17.

LEMMA 4.16. Let $X$ be a continuum, let $n$ be an integer greater than or equal to two, and let $f: X \rightarrow X$ be a surjective map. Then the following are equivalent:

(1) $\operatorname{per}(f)$ is dense in $X$;

(2) $\operatorname{per}\left(\mathcal{F}_{n}(f)\right)$ is dense in $\mathcal{F}_{n}(X)$;

(3) $\operatorname{per}\left(\mathcal{S F}_{n}(f)\right)$ is dense in $\mathcal{S F}_{n}(X)$.

Proof. By [11, Theorem 4.7], we obtain that (1) and (2) are equivalent and, by Lemma 3.3, we have that (2) implies (3).

Suppose that $\operatorname{per}\left(\mathcal{S F}_{n}(f)\right)$ is dense in $\mathcal{S F}_{n}(X)$, we see that $\operatorname{per}\left(\mathcal{F}_{n}(f)\right)$ is dense in $\mathcal{F}_{n}(X)$. Let $\mathcal{U}$ be a nonempty open subset of $\mathcal{F}_{n}(X)$. We prove that $\mathcal{U} \cap \operatorname{per}\left(\mathcal{F}_{n}(f)\right) \neq \emptyset$. In other words, we see that there exist $A \in \mathcal{U}$ 
and $k \in \mathbb{N}$ such that $\left(\mathcal{F}_{n}(f)\right)^{k}(A)=A$. By [11, Lemma 4.2], there exist nonempty open subsets $U_{1}, U_{2}, \ldots, U_{n}$ of $X$ such that $\left\langle U_{1}, U_{2}, \ldots, U_{n}\right\rangle_{n} \subseteq \mathcal{U}$. For each $i \in\{1,2, \ldots, n\}$, let $W_{i}$ be a nonempty open subset of $X$ such that $W_{i} \subseteq U_{i}$ and for each $i, j \in\{1,2, \ldots, n\}, W_{i} \cap W_{j} \neq \emptyset$, if $i \neq j$. It follows that $\left\langle W_{1}, W_{2}, \ldots, W_{n}\right\rangle_{n}$ is a nonempty open subset of $\mathcal{F}_{n}(X)$ such that:

$$
\left\langle W_{1}, W_{2}, \ldots, W_{n}\right\rangle_{n} \subseteq\left\langle U_{1}, U_{2}, \ldots, U_{n}\right\rangle_{n} \subseteq \mathcal{U}
$$

and

$$
\left\langle W_{1}, W_{2}, \ldots, W_{n}\right\rangle_{n} \cap \mathcal{F}_{1}(X)=\emptyset .
$$

By Remark 2.2, we have that $q\left(\left\langle W_{1}, W_{2}, \ldots, W_{n}\right\rangle_{n}\right)$ is a nonempty open subset of $\mathcal{S F}_{n}(X)$ such that $F_{X} \notin q\left(\left\langle W_{1}, W_{2}, \ldots, W_{n}\right\rangle_{n}\right)$. Since $\operatorname{per}\left(\mathcal{S F}_{n}(f)\right)$ is dense in $\mathcal{S F}_{n}(X)$, there exist $A \in\left\langle W_{1}, W_{2}, \ldots, W_{n}\right\rangle_{n}$ and $k \in \mathbb{N}$ such that $\left(\mathcal{S F}_{n}(f)\right)^{k}(q(A))=q(A)$. By Proposition $4.1(\mathrm{~b}), q\left(\left(\mathcal{F}_{n}(f)\right)^{k}(A)\right)=q(A)$. Furthermore, by Remark $2.2,\left(\mathcal{F}_{n}(f)\right)^{k}(A)=A$. Hence, $\mathcal{U} \cap \operatorname{per}\left(\mathcal{F}_{n}(f)\right) \neq \emptyset$. Therefore, $\operatorname{per}\left(\mathcal{F}_{n}(f)\right)$ is dense in $\mathcal{F}_{n}(X)$.

TheOREM 4.17. Let $X$ be a continuum, let $n$ be an integer greater than or equal to two, and let $f: X \rightarrow X$ be a map. Then the following are equivalent:

(1) $f$ is chaotic and weakly mixing;

(2) $\mathcal{F}_{n}(f)$ is chaotic;

(3) $\mathcal{S F}_{n}(f)$ is chaotic.

Proof. By [11, Theorem 4.9], we have that (1) and (2) are equivalent. On the other hand, by Theorem 4.10 and by Lemma 4.16 , we conclude that (2) and (3) are equivalent.

THEOREM 4.18. Let $X$ be a nondegenerate continuum, let $n$ be an integer greater than or equal to two, and let $f: X \rightarrow X$ be a map. Consider the following statements:

(1) $f$ is minimal;

(2) $\mathcal{F}_{n}(f)$ is minimal;

(3) $\mathcal{S F}_{n}(f)$ is minimal.

Then (2) implies (3), (3) implies (1), (2) implies (1), (1) does not imply (2) and (1) does not imply (3).

Proof. By Theorem 3.4, we have that (2) implies (3).

Suppose that $\mathcal{S F}_{n}(f)$ is minimal, we see that $f$ is minimal. For this end, let $x \in X$ and let $U$ be a nonempty open subset of $X$. We consider nonempty open subsets $V_{1}$ and $V_{2}$ of $X$ such that $V_{1} \cup V_{2} \subseteq U$ and $V_{1} \cap V_{2}=\emptyset$. Hence, $\left\langle V_{1}, V_{2}\right\rangle_{n}$ is a nonempty open subset of $\mathcal{F}_{n}(X)$ such that $\left\langle V_{1}, V_{2}\right\rangle_{n} \cap \mathcal{F}_{1}(X)=\emptyset$. By Remark 2.2, $q\left(\left\langle V_{1}, V_{2}\right\rangle_{n}\right)$ is a nonempty open subset of $\mathcal{S F}_{n}(X)$. Note that $F_{X} \notin q\left(\left\langle V_{1}, V_{2}\right\rangle_{n}\right)$. Let $y \in X \backslash\{x\}$ and we consider $A=\{x, y\}$. Clearly $A \in \mathcal{F}_{n}(X) \backslash \mathcal{F}_{1}(X)$. Since $\mathcal{S F}_{n}(f)$ is minimal, $\operatorname{orb}\left(q(A), \mathcal{S} \mathcal{F}_{n}(f)\right)$ is dense in $\mathcal{S F}_{n}(X)$. Thus, $q\left(\left\langle V_{1}, V_{2}\right\rangle_{n}\right) \cap \operatorname{orb}\left(q(A), \mathcal{S F}_{n}(f)\right) \neq \emptyset$. Let $C \in\left\langle V_{1}, V_{2}\right\rangle_{n}$ 
and let $k \in \mathbb{N} \cup\{0\}$ such that $\left(\mathcal{S F}_{n}(f)\right)^{k}(q(A))=q(C)$. By Proposition 4.1 (b), $q\left(\left(\mathcal{F}_{n}(f)\right)^{k}(A)\right)=q(C)$. Hence, by Remark $2.2,\left(\mathcal{F}_{n}(f)\right)^{k}(A)=C$. Thus, by Proposition 4.1 (a), $f^{k}(A)=C$. Since $x \in A$ and $C \subseteq U$, it follows that $f^{k}(x) \in U$. In consequence, $U \cap \operatorname{orb}(x, f) \neq \emptyset$. This implies that $f$ is minimal.

Since (2) implies (3) and (3) implies (1), we have that (2) implies (1).

Finally, in Example 4.6, we obtain that (1) does not implies (2) and that (1) does not implies (3).

Note that as a consequence of Diagram 1 and Theorem 4.10, we obtain that.

COROllary 4.19. Let $X$ be a nondegenerate continuum, let $n$ be an integer greater than or equal to two, and let $f: X \rightarrow X$ be a map. If $\mathcal{S F}_{n}(f)$ is minimal, then $\mathcal{F}_{n}(f)$ is transitive.

Moreover, by Corollary 4.19 and Theorem 4.11, we obtain:

Corollary 4.20. Let $X$ be a nondegenerate continuum, let $n$ be an integer greater than or equal to two, and let $f: X \rightarrow X$ be a map. If $\mathcal{S F}_{n}(f)$ is minimal, then $f, \mathcal{F}_{n}(f)$ and $\mathcal{S F}_{n}(f)$ are weakly mixing.

QUESTION 4.2. Let $X$ be a continuum, let $n$ be an integer greater than or equal to two, and let $f: X \rightarrow X$ be a map. If $\mathcal{S F}_{n}(f)$ is minimal, then is $\mathcal{F}_{n}(f)$ minimal?

Recall that $f: X \rightarrow X$ is an open map if for each open subset $A$ in $X$, $f(A)$ is an open subset in $X$.

Corollary 4.21. Let $X$ be a nondegenerate continuum, let $n$ be an integer greater than or equal to two, and let $f: X \rightarrow X$ be a map. If $\mathcal{F}_{n}(f)$ is minimal and open, then $f$ is a homeomorphism.

Proof. The result follows from Theorem 4.18, [4, Theorem 5.3] and [14, Theorem $2.4(2)]$.

\section{Other DYNAMiCAL PROPERTIES}

We begin this section with the following result.

THEOREM 5.1. Let $X$ be a nondegenerate continuum, let $n$ be an integer greater than or equal to two, and let $f: X \rightarrow X$ be a map. Consider the following statements:

(1) $f$ is irreducible;

(2) $\mathcal{F}_{n}(f)$ is irreducible;

(3) $\mathcal{S F}_{n}(f)$ is irreducible.

Then (2) implies (1) and (3) implies (1). 
Proof. Suppose that $\mathcal{F}_{n}(f)$ is irreducible, we prove that $f$ is irreducible. Let $A$ be a nonempty closed subset of $X$ such that $f(A)=X$. It follows that $\langle A\rangle_{n}$ is a nonempty closed subset of $\mathcal{F}_{n}(X)$ such that $\mathcal{F}_{n}(f)\left(\langle A\rangle_{n}\right)=\mathcal{F}_{n}(X)$. Since $\mathcal{F}_{n}(f)$ is irreducible, we have that $\langle A\rangle_{n}=\mathcal{F}_{n}(X)$. Thus, $\mathcal{F}_{1}(X) \subseteq\langle A\rangle_{n}$. This implies that $X=A$. Hence, $f$ is irreducible.

Now, suppose that $\mathcal{S} \mathcal{F}_{n}(f)$ is irreducible, we prove that $f$ is irreducible. Let $A$ be a nonempty closed subset of $X$ such that $f(A)=X$. It follows that $\langle A\rangle_{n}$ is a nonempty closed subset of $\mathcal{F}_{n}(X)$ such that $\mathcal{F}_{n}(f)\left(\langle A\rangle_{n}\right)=\mathcal{F}_{n}(X)$. Hence, $q\left(\mathcal{F}_{n}(f)\left(\langle A\rangle_{n}\right)\right)=\mathcal{S F}_{n}(X)$. In consequence, by Proposition $4.1(\mathrm{~b})$, $\mathcal{S F}_{n}(f)\left(q\left(\langle A\rangle_{n}\right)\right)=\mathcal{S F}_{n}(X)$. Since $q\left(\langle A\rangle_{n}\right)$ is a nonempty closed subset of $\mathcal{S F}_{n}(X)$ and $\mathcal{S} \mathcal{F}_{n}(f)$ is irreducible, we have that $q\left(\langle A\rangle_{n}\right)=\mathcal{S F}_{n}(X)$. Now, let $x \in X$ and let $y \in X \backslash\{x\}$. Let $B=\{x, y\}$. Since $q(B) \in \mathcal{S F}_{n}(X)$, there exists $C \in\langle A\rangle_{n}$ such that $q(C)=q(B)$. By Remark 2.2, $C=B$. Hence, $x \in A$. In consequence, $X=A$. Therefore, $f$ is irreducible.

COROllary 5.2. Let $X$ be a nondegenerate continuum, let $n$ be an integer greater than or equal to two, and let $f: X \rightarrow X$ be a map. Then $\mathcal{F}_{n}(f)$ is irreducible and open if and only if $f$ is a homeomorphism.

Proof. The corollary follows easily from Theorem 5.1, [4, Theorem 5.3] and [14, Lemma 2.2].

Note that feebly open maps are also know semi-open maps in the literature. The following result is [6, Theorem 10.1].

TheOREM 5.3. Let $X$ be a continuum, let $n$ be an integer greater than or equal to two, and let $f: X \rightarrow X$ be a surjective map. Then the following are equivalent:

(1) $f$ is feebly open;

(2) $\mathcal{F}_{n}(f)$ is feebly open;

(3) $\mathcal{S F}_{n}(f)$ is feebly open.

Using the Diagram 1 and Theorem 5.3, we obtain the following result.

Corollary 5.4. Let $X$ be a continuum, let $n$ be an integer greater than or equal to two, and let $f: X \rightarrow X$ be a map. Then the following statements hold.

(1) If $f$ is irreducible, then $\mathcal{F}_{n}(f)$ is feebly open.

(2) If $f$ is irreducible, then $\mathcal{S F}_{n}(f)$ is feebly open.

(3) If $\mathcal{F}_{n}(f)$ is irreducible, then $\mathcal{S F}_{n}(f)$ is feebly open.

(4) If $\mathcal{S F}_{n}(f)$ is irreducible, then $\mathcal{F}_{n}(f)$ is feebly open.

Questions 5.5. Let $X$ be a continuum, let $n$ be an integer greater than or equal to two, and let $f: X \rightarrow X$ be a map.

(1) If $f$ is irreducible, then is $\mathcal{F}_{n}(f)$ irreducible?

(2) If $f$ is irreducible, then is $\mathcal{S F}_{n}(f)$ irreducible? 
(3) If $\mathcal{F}_{n}(f)$ is irreducible, then is $\mathcal{S F}_{n}(f)$ irreducible?

(4) If $\mathcal{S F}_{n}(f)$ is irreducible, then is $\mathcal{F}_{n}(f)$ irreducible?

TheOREM 5.6. Let $X$ be a nondegenerate continuum, let $n$ be an integer greater than or equal to two, and let $f: X \rightarrow X$ be a surjective map. Consider the following statements:

(1) $f$ is turbulent;

(2) $\mathcal{F}_{n}(f)$ is turbulent;

(3) $\mathcal{S F}_{n}(f)$ is turbulent.

Then (1) implies (2) and (3).

Proof. Suppose that $f$ is turbulent, we prove that $\mathcal{S F}_{n}(f)$ is turbulent. Let $K$ and $C$ be nondegenerate compact subsets of $X$ such that $K \cap C$ has at most one point and $K \cup C \subseteq f(K) \cap f(C)$. It is easy to see that $\langle K\rangle_{n}$ and $\langle C\rangle_{n}$ are nondegenerate compact subsets of $\mathcal{F}_{n}(X)$. Let $\Lambda=q\left(\langle K\rangle_{n}\right)$ and $\Gamma=q\left(\langle C\rangle_{n}\right)$. It follows that $\Lambda$ and $\Gamma$ are nondegenerate compact subsets of $\mathcal{S F}_{n}(X)$.

We show that $\Lambda \cap \Gamma$ has at most one point. For this, note that if $K \cap C=\emptyset$, then $\langle K\rangle_{n} \cap\langle C\rangle_{n}=\emptyset$. Since $\mathcal{F}_{1}(K) \subseteq\langle K\rangle_{n}$ and $\mathcal{F}_{1}(C) \subseteq\langle C\rangle_{n}$, it follows that $F_{X} \in \Lambda \cap \Gamma$.

On the other hand, if $K \cap C=\{a\}$, we obtain that $\langle K\rangle_{n} \cap\langle C\rangle_{n}=$ $\{\{a\}\}$. Thus, $F_{X} \in \Lambda \cap \Gamma$. Now, if $\chi \in(\Lambda \cap \Gamma) \backslash\left\{F_{X}\right\}$, then there exist $A \in\langle K\rangle_{n} \backslash \mathcal{F}_{1}(X)$ and $B \in\langle C\rangle_{n} \backslash \mathcal{F}_{1}(X)$ such that $q(A)=\chi=q(B)$. By Remark 2.2, $A=B$. This proves that $A \subseteq K \cap C$. Thus, $K \cap C$ has at least two elements, which is a contradiction. Therefore, $\Lambda \cap \Gamma$ has at most one point.

Now, we see that $\Lambda \cup \Gamma \subseteq \mathcal{S F}_{n}(f)(\Lambda) \cap \mathcal{S F}_{n}(f)(\Gamma)$. Let $\chi \in \Lambda \cup \Gamma$. Hence, there exits $A \in\langle K\rangle_{n} \cup\langle C\rangle_{n}$ such that $q(A)=\chi$. Since $A \subseteq$ $K \cup C$ and $K \cup C \subseteq f(K) \cap f(C)$, we have that $A \subseteq f(K) \cap f(C)$. This implies that $A \in\langle f(K) \cap f(C)\rangle_{n}$. Thus, $A \in\langle f(K)\rangle_{n} \cap\langle f(C)\rangle_{n}$. In consequence, $q(A) \in q\left(\langle f(K)\rangle_{n}\right) \cap q\left(\langle f(C)\rangle_{n}\right)$. Since $q(A)=\chi$, we have that $\chi \in q\left(\mathcal{F}_{n}(f)\left(\langle K\rangle_{n}\right)\right) \cap q\left(\mathcal{F}_{n}(f)\left(\langle C\rangle_{n}\right)\right)$. By Proposition 4.1 (b), $\chi \in \mathcal{S F}_{n}(f)\left(q\left(\langle K\rangle_{n}\right)\right) \cap \mathcal{S F}_{n}(f)\left(q\left(\langle C\rangle_{n}\right)\right)$. It follows that $\chi \in \mathcal{S F}_{n}(f)(\Lambda) \cap$ $\mathcal{S F}_{n}(f)(\Gamma)$. In consequence, $\Lambda \cup \Gamma \subseteq \mathcal{S F}_{n}(f)(\Lambda) \cap \mathcal{S F}_{n}(f)(\Gamma)$. Therefore, $\mathcal{S F}_{n}(f)$ is turbulent.

The proof (1) implies (2) is similar to the proof (1) implies (3).

We end this paper with following questions.

QUeSTIONS 5.7. Let $X$ be a continuum, let $n$ be an integer greater than or equal to two, and let $f: X \rightarrow X$ be a map.

(i) If $\mathcal{F}_{n}(f)$ is turbulent, then is $f$ turbulent?

(ii) If $\mathcal{F}_{n}(f)$ is turbulent, then is $\mathcal{S F}_{n}(f)$ turbulent?

(iii) If $\mathcal{S F}_{n}(f)$ is turbulent, then is $f$ turbulent?

(iv) If $\mathcal{S F}_{n}(f)$ is turbulent, then is $\mathcal{F}_{n}(f)$ turbulent? 


\section{REFERENCES}

[1] G. Acosta, A. Illanes and H. Méndez-Lango, The transitivity of induced maps, Topology Appl. 156 (2009), 1013-1033.

[2] J. Banks, Chaos for induced hyperspace maps, Chaos Solitons Fractals 25 (2005), $681-685$.

[3] F. Barragán, On the $n$-fold symmetric product suspensions of a continuum, Topology Appl. 157 (2010), 597-604.

[4] F. Barragán, Induced maps on $n$-fold symmetric product suspensions, Topology Appl. 158 (2011), 1192-1205.

[5] F. Barragán, Aposyndetic properties of the $n$-fold symmetric product suspensions of a continuum, Glas. Mat. Ser. III 49(69) (2014), 179-193.

[6] F. Barragán, S. Macías and J. F. Tenorio, More on induced maps on $n$-fold symmetric product suspensions, Glas. Mat. Ser. III 50(70) (2015), 489-512.

[7] J. Camargo, C. García and A. Ramírez, Transitivity of the induced map $C_{n}(f)$, Rev. Colombiana Mat. 48 (2014), 235-245.

[8] J. S. Cánovas-Peña and G. S. López, Topological entropy for induced hyperspace maps, Chaos Solitons Fractals 28 (2006), 979-982.

[9] J. Dugundji, Topology, Allyn and Bacon, Inc., Boston, 1966.

[10] J. L. Gómez-Rueda, A. Illanes and H. Méndez-Lango, Dynamic properties for the induced maps in the symmetric products, Chaos Solitons Fractals 45 (2012), 11801187.

[11] G. Higuera and A. Illanes, Induced mappings on symmetric products, Topology Proc. 37 (2011), 367-401.

[12] R. Gu and W. Guo, On mixing property in set-valued discrete systems, Chaos Solitons Fractals 28 (2006), 747-754.

[13] A. Illanes and S. B. Nadler, Jr., Hyperspaces. Fundamentals and recent advances, Marcel Dekker, New York, 1999.

[14] S. Kolyada, L. Snoha and S. Trofimchuk, Noninvertible minimal maps, Fund. Math. 168 (2001), 141-163.

[15] D. Kwietniak and M. Misiurewicz, Exact Devaney chaos and entropy, Qual. Theory Dyn. Syst. 6 (2005), 169-179.

[16] X. Ma, B. Hou and G. Liao, Chaos in hyperspace system, Chaos Solitons Fractals 40 (2009), 653-660

[17] S. Macías, Topics on continua, Chapman \& Hall/CRC, Boca Raton, 2005.

[18] S. B. Nadler, Jr., Hyperspaces of sets, Marcel Dekker, New York-Basel, 1978. Reprinted by: Sociedad Matemática Mexicana, México, 2006.

[19] S. B. Nadler, Jr., Continuum theory. An introduction, Marcel Dekker, New York, 1992.

[20] A. Peris, Set-valued discrete chaos, Chaos Solitons Fractals 26 (2005), 19-23.

[21] H. Román-Flores, A note on transitivity in set-valued discrete systems, Chaos Solitons Fractals 17 (2003), 99-104.

[22] M. Sabbaghan and H. Damerchiloo, A note on periodic points and transitive maps, Math. Sci. Q. J. 5 (2011), 259-266.

[23] Y. Wang and G. Wei, Characterizing mixing, weak mixing and transitivity of induced hyperspace dynamical systems, Topology Appl. 155 (2007), 56-68. 
F. Barragán

Instituto de Física y Matemáticas

Universidad Tecnológica de la Mixteca

Carretera a Acatlima, Km. 2.5, Huajuapan de León, Oaxaca, C.P. 69000

México

E-mail: franco@mixteco.utm.mx

A. Santiago-Santos

Instituto de Física y Matemáticas

Universidad Tecnológica de la Mixteca

Carretera a Acatlima, Km. 2.5, Huajuapan de León, Oaxaca, C.P. 69000

México

E-mail: alicia@mixteco.utm.mx

J. F. Tenorio

Instituto de Física y Matemáticas

Universidad Tecnológica de la Mixteca

Carretera a Acatlima, Km. 2.5, Huajuapan de León, Oaxaca, C.P. 69000

México

E-mail: jtenorio@mixteco.utm.mx

Received: 3.6.2015.

Revised: 19.1.2016. 